\title{
Index to Volume 16, 2014
}

\begin{tabular}{|c|c|c|c|c|c|}
\hline A & & Camp PG & $467-76$ & Er F & 65 \\
\hline Ackery A & $243-46$ & Campbell SG & 12 & Evans C & $207-13$ \\
\hline Affleck A & $1-7$ & Carleton B & $467-76$ & & \\
\hline Afilalo M & $193-206$ & Carlson JN & $378-82$ & $\mathbf{F}$ & \\
\hline Ahn JS & 393-404 & Carr D & $421-24,425-28$ & Fagnoul D & $504-7$ \\
\hline Ali S & $352-60,449-57$ & Carrière B & $281-87$ & Fergusson D & $69-79$ \\
\hline Allain-Boulé N & $136-43$ & Castelino $\mathrm{T}$ & $193-206$ & Fleet R & $437-48$ \\
\hline Alsakha A & $327-29$ & Caudle J & $220-28$ & Foerster CR & 10 \\
\hline Andrew MK & $370-77$ & Chambers A & $352-60$ & Frank JR & $345-51$ \\
\hline Andrews L & 214-19 & Champlain F & 94-105 & Frass $\mathrm{M}$ & 9 \\
\hline Archambault P & $437-48$ & Chan T & $485-89,490-93$ & Fratu R-F & $136-43$ \\
\hline Arora S & $214-19$ & Chauny J-M & $136-43$ & Frechette $M$ & $25-33$ \\
\hline Atkinson P & $265-68,269-72$ & Chen EM & $502-3$ & Froese P & 12 \\
\hline Audette L-D & $437-48$ & Cheng A & $383-92$ & Frost DW & $164-70$ \\
\hline Audette-Côté J-S & 229-42 & Cheng C-J & 344 & & \\
\hline & & Chenkin J & $164-70$ & G & \\
\hline B & & Chiang W-F & $247-51$ & Gassanov N & 65 \\
\hline Backer D de & $504-7$ & Christenson J & 106-19 & Geerts W & $53-62$ \\
\hline Baranchuk A & $330-33$ & Cleiman P & $458-66$ & Genuis ED & $405-10$ \\
\hline Baw B & 490-93 & Clément K & 281-87 & Gin K & 106-19 \\
\hline Belanger P & $220-28$ & Colacone A & $193-206$ & Goh E & $411-12,502-3$ \\
\hline Bellamy MA & $63-64$ & Croskerry P & 13-19 & Goldman RD & $467-76$ \\
\hline Berdahl C, & $288-95$ & Curtis SJ & $352-60$ & Goldstein J & $370-77$ \\
\hline Bergeron É & $136-43$ & Cwinn AA & 259-64 & Goubella A & 504-7 \\
\hline Bhayana R & $288-95$ & & & Grafstein E 4 & $106-19,185-92$ \\
\hline Bilbey J & $131-35$ & & & Grant V & 383-92 \\
\hline Bleskie D & $69-79$ & D & & Gravel J & $281-87$ \\
\hline Bogner V & 494-96 & Dankoff J 151-5 & $155-57,193-206$ & Green R & $69-79$ \\
\hline Boivin J-F & $94-105$ & Day GS & $164-70$ & Grock A & 214-19 \\
\hline Boothroyd LJ & $94-105$ & Deady B & $160-63$ & Guimont C & $136-43$ \\
\hline Botnaru $T$ & $151-54$ & DeJong P & $330-33$ & Guttman A & $327-29$ \\
\hline Boychuk B & $106-19$ & Delaney JS & $497-501$ & & \\
\hline Brayman C & $485-89$ & D'Eon M & 273-80 & $\mathbf{H}$ & \\
\hline Bridge J & $207-13$ & Devon J & 84-87 & Haasters F & 494-96 \\
\hline Brimioulle $\mathrm{S}$ & $504-7$ & Dhanoa D & $334-38$ & Haggar C & $323-26$ \\
\hline Brison RJ & $25-33$ & Diksic D & $304-13$ & Hall C & $171-76$ \\
\hline Brnjac E & $53-62$ & Doan Q & $405-10$ & Hayward G & 214-19 \\
\hline Bromley M & $296-303$ & Donnon T & $144-50$ & Heathcote S & $467-76$ \\
\hline Brooks SC & 314-21 & Dowdall H & $25-33$ & Heiner JD & $158-59$ \\
\hline Brunner NW & $41-52$ & Drendel AL & $449-57$ & Helfen T & $494-96$ \\
\hline Buick JE & $314-21$ & Dreyer JF & $393-404$ & Hellings CR & $288-95$ \\
\hline Bullard MJ & $485-89$ & Dufresne $M$ & $136-43$ & Henneberry $\mathrm{R}$ & $265-68$ \\
\hline Burns T & $80-83$ & Dulai S & $449-57$ & $\begin{array}{l}\text { Henneberry } \\
\text { Hess EP }\end{array}$ & $120-30$ \\
\hline Burry L & $252-56$ & & & Heyd C & $171-76$ \\
\hline Burton KR & $334-38$ & E & & Hildebrand K & $296-303$ \\
\hline Butler C & $171-76$ & Eby D & 8 & Hill S & 20-24 \\
\hline & & Edmonds ML & $393-404$ & Hiscox C & $296-303$ \\
\hline C & & Eisenberg MJ & $94-105$ & Hodges D & 160-63 \\
\hline Calder LA & 259-64 & Émond M & $136-43,229-42$ & Hohl C & $177-82$ \\
\hline Camilion M & 214-19 & Eppich W & 383-92 & Howey E & $34-40$ \\
\hline
\end{tabular}




\begin{tabular}{|c|c|c|c|c|c|}
\hline Hubbard R & $370-77$ & McEachern JD & $361-69$ & Puchalski Ritchie L & $458-66$ \\
\hline Hunchak C & $458-66$ & McFaull S & $25-33$ & Pullen B & $160-63$ \\
\hline Hutton B & $69-79$ & McGillivray D & $304-13$ & & \\
\hline \multirow[t]{2}{*}{ Huynh T } & $94-105$ & McIntyre L & 69-79 & $\mathbf{R}$ & \\
\hline & & McLeod SL & $393-404$ & Rac VE & $314-21$ \\
\hline I & & Menchine $M$ & $214-19$ & Ramanathan K & $41-52$ \\
\hline Ingber S & $53-62$ & Menegazzi JJ & $378-82$ & Reid SM & 183 \\
\hline \multirow[t]{2}{*}{ Innes G } & $106-19,185-92$ & Menezes RG & 11,436 & Rigby I & $144-50$ \\
\hline & & Meshkat N & $458-66$ & Ring $\mathrm{T}$ & $131-35$ \\
\hline $\mathbf{J}$ & & Michael K & $330-33$ & Robak O & \\
\hline Jama S & $477-84$ & Mikrogianakis A & $383-92$ & Robichaud L & $66-68$ \\
\hline Jayaraman S & 343,436 & Miller P & $84-87$ & Robinson T & $383-92$ \\
\hline Jena NN & 343,436 & Misra S & $458-66$ & Rockwood K & $370-77$ \\
\hline Johnson DW & $352-60$ & Mitchell IV & $131-35$ & Rosenberg H & 322 \\
\hline Jones GL & $339-42$ & Mohindra R & $323-26$ & Ross P & $265-68$ \\
\hline \multirow[t]{2}{*}{ Joseph L } & $94-105$ & Mohr KL & $361-69$ & Rowe $\mathrm{BH}$ & $185-92$ \\
\hline & & Mok E & $304-13$ & & \\
\hline $\mathbf{K}$ & & Moore K & $220-28$ & $\mathbf{S}$ & \\
\hline Kanz KG & 494-96 & Moore L & $136-43$ & Salcido DD & $378-82$ \\
\hline Kassam A & $144-50$ & house P & $370-77$ & SS & $66-68$ \\
\hline Kavsak P & $20-24$ & $\mathrm{TL}$ & $158-59$ & Sandhu N & $383-92$ \\
\hline Kester-Greene N & $414-20$ & Morrison LJ & $184,314-21$ & Scheuermeyer FX & $41-52,106-19$ \\
\hline Khan Y & $458-66$ & Mottillo S & $497-501$ & Schull MJ & $288-95$ \\
\hline Kierzek G & $314-21$ & Mullan PC & 435 & Segal E & $94-105$ \\
\hline Kircher J & $449-57$ & Musgrave E & $485-89$ & Selby R & $53-62$ \\
\hline Kissoon N & $429-34$ & Mutschler W & 494-96 & Senthilkumaran S & $11,343,436$ \\
\hline Kukaswadia A & $25-33$ & & & Shah S & 11 \\
\hline Kulasegaram K & $34-40$ & & & Shajari S & $467-76$ \\
\hline \multirow[t]{2}{*}{ Kuuskne M } & $155-57$ & $\mathbf{N}$ & & Shamji AI & $164-70$ \\
\hline & & Nagji A & $508-9$ & Sharma A & 413 \\
\hline $\mathbf{L}$ & & Nathens AB & $207-13$ & Sherbino J & $34-40$ \\
\hline LaMothe J & $296-303$ & Newton AS & $352-60,449-57$ & Singh D & 413 \\
\hline Lang E & 185-92 & Nguyen V & 94-105 & Smith MA & $467-76$ \\
\hline Lapinsky S & $252-56$ & Nia AM & 65 & ni $R$ & $330-33$ \\
\hline Lavoie A & $136-43$ & Nolan B & $184,425-28$ & $\mathrm{~N}$ & $193-206$ \\
\hline Le Sage N & $136-43$ & Norman G & $34-40$ & $\mathrm{LE}$ & 435 \\
\hline Lee AC & $345-51$ & Norton SP & $467-76$ & $\mathrm{k}$ & $193-206$ \\
\hline Lee JS & $53-62,414-20$ & & & pien JE & $361-69$ \\
\hline Lee PA & 185-92 & $\mathbf{O}$ & & Stiell IG & $120-30,477-84$ \\
\hline Leswick DA & $361-69$ & Oakes I & $131-35$ & Stoneham GW & $361-69$ \\
\hline Letovsky E & $1-7$ & O'Connell KI & 435 & Strote J & $80-83$ \\
\hline Li Q & $288-95$ & Ovens H & $1-7$ & Suffoletto BP & $378-82$ \\
\hline Lin $S$ & $314-21$ & Owen II & $20-24$ & Swartz RH & $164-70$ \\
\hline Lin S-H & $247-51,344$ & & & & \\
\hline Little A & $252-56$ & & & $\mathbf{T}$ & \\
\hline Lockwood JR & $243-46,421-24$ & $\mathbf{P}$ & & Taccone FS & $504-7$ \\
\hline Logue ES & $378-82$ & Paolucci EO & $296-303$ & Tallon JM & $207-13$ \\
\hline \multirow[t]{2}{*}{ Lorette J } & $69-79$ & Patel B & 435 & \multicolumn{2}{|c|}{ Thiruganasambandamoorthy $\mathrm{V}$} \\
\hline & & Perry JJ & $120-30$ & & $120-30$ \\
\hline M & & Pickett $W$ & $25-33$ & Thirumalaikolundus & Isubramanian \\
\hline Maher PJ & $80-83$ & Plenk K & $425-28$ & $\mathrm{P}$ & $11,343,436$ \\
\hline Malo C & $229-42$ & Plourde M & $136-43$ & Thompson W & $25-33$ \\
\hline Marcoux J & $437-48$ & Poitras J & $437-48$ & Thooft A & $504-7$ \\
\hline McAleer S & $273-80$ & Probst MA & 214-19 & Tomich EB & $63-64$ \\
\hline
\end{tabular}




$\begin{array}{lrlrlr}\text { Tourigny-Ruel G } & 304-13 & \text { Vandermeer B } & \text { 352-60, 449-57 } & \text { Williamson J } & 352-60 \\ \text { Tozer AP } & 220-28 & \text { Vanier L } & 136-43 & \text { Williamson LD } & 131-35 \\ \text { Travers A } & 370-77 & \text { Vermeulen MJ } & 288-95 & \text { Wojtowicz JM } & 339-42 \\ \text { Trinder K } & 273-80 & \text { Villa J } & 437-48 & \text { Wong H } & 106-19 \\ \text { Truong C } & 252-56 & \text { Vilneff R } & 185-92 & \text { Woo MY } & 345-51 \\ \text { Tung D } & 252-56 & \text { Vincent J-L } & 504-7 & \text { Woods RA } & 273-80 \\ \text { Turgeon AF } & 229-42 & \text { Vukusic A } & 131-35 & \text { Worrall JC } & 477-84 \\ \text { Turko E } & 120-30 & & & \text { Worster A } & 20-24 \\ & & \text { W } & & & \\ \text { U } & & \text { Waines BM } & 20-24 & \text { X } & 193-206 \\ \text { Unger B } & 485-89 & \text { Walsh M } & 80-83 & \text { Xue X } \\ \text { Upadhye S } & 490-93 & \text { Wang C-C } & 247-51 & & \\ \text { Uyeki T } & 429-34 & \text { Wang D } & 185-92 & \text { Y } & 171-76 \\ & & \text { Wang X } & 334-38 & \text { Yarema M } & 247-51 \\ \text { V } & & \text { Wanke-Jellinek L } & 494-96 & \text { Yar } & 322 \\ \text { Vadeboncoeur A } & 94-105 & \text { Ward J } & 20-24 & \text { Yeh F-C } & 405-10 \\ \text { van Rheenen S } & 185-92 & \text { Warren D } & 485-89 & \text { Yoo JH } & 106-19 \\ \text { Van Zyl HP } & 131-35 & \text { Wells GA } & 120-30 & \text { Yu A } & \text { Yu E } \\ \text { VandenBerg SD } & 257-58 & \text { White N } & 296-303 & & \end{array}$

